\title{
ESTIMATION OF SENSITIVE MATERIAL PROPERTIES OF AN OPEN HOLE TENSION TEST OF COMPOSITE LAMINATES
}

\author{
A. Sasikumar ${ }^{1 *}$, I.R. Cózar ${ }^{1}$, O. Vallmajó ${ }^{1}$, S. Abdel-Monsef ${ }^{1}$, M. De Lozzo ${ }^{2}$, A. Turon ${ }^{1}$ \\ ${ }^{1}$ AMADE, Polytechnic School, Universitat de Girona, Campus Montilivi s/n, 17073 Girona, Spain \\ ${ }^{2}$ IRT Saint Exupéry, CS34436, 3 Rue Tarfaya. 31400 Toulouse, France \\ * corresponding.author: aravind.sasikumar@udg.edu
}

Sensitivity analysis (SA) is multi-disciplinary and is applied across various fields to estimate the sensitive parameters that influence a target output response [1,2]. In the context of composite materials, the failure strength of a laminate depends on numerous material properties such as the elastic, strength and fracture properties of both the fiber and the matrix system. Hence, integrating a SA to a composite laminate test helps to screen the influential and non-influential material properties, thus leading to potential benefits. Once the sensitive parameters are identified, the experimental tests to obtain these corresponding material properties can be performed with more care, making sure that the associated uncertainly is minimal. Further within the framework of uncertainty quantification, these selected key properties can be well characterized by increasing the number of specimens to obtain a better statistical distribution. In addition, identification of the sensitive material properties can help in finite element based optimization methods and in the generation of metamodels, where only the key parameters are considered to optimize the failure strength. These numerous benefits substantially support the importance of performing SA within the context of composite materials.

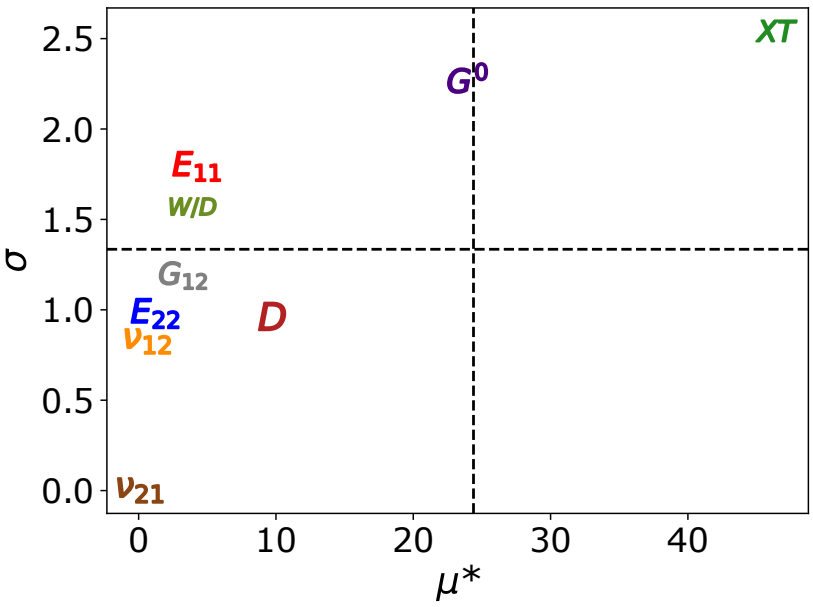

(a)

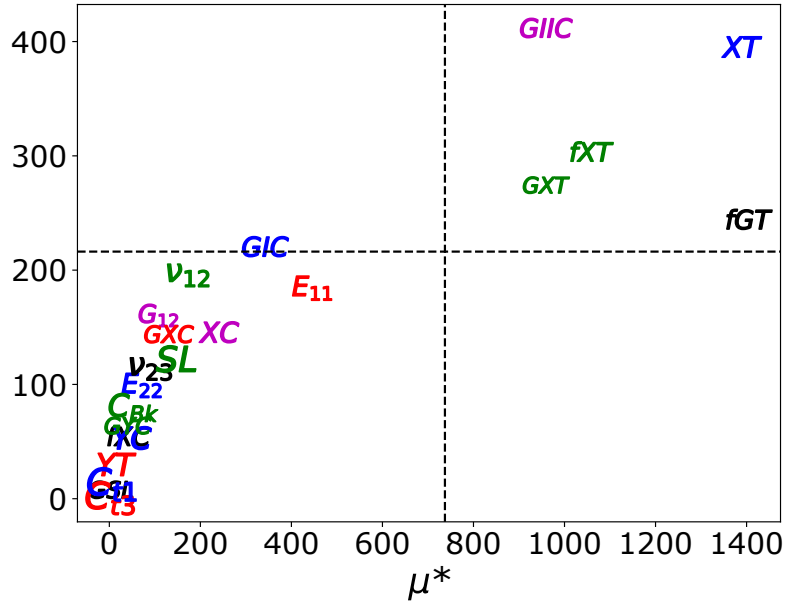

(b)

Figure 1: Morris sensitivity analysis results showing the sensitive material properties of an open hole tensile test from (a) analytical and (b) FEM numerical methods, respectively. (Higher $\mu^{*}$ represents higher total order effect and higher $\sigma$ represents higher interaction effects)

In this work, we selected an open hole tension (OHT) test case of a quasi-isotropic laminate, and performed a SA using analytical and numerical FEM based methods. Morris One-Ata-Time (MOAT) sensitivity analysis [4] was selected after a case study due to its relatively high accuracy with reduced computational effort. MOAT method provides the total and the 
interaction effect of a parameter from the modified mean $\left(\mu^{*}\right)$ and standard deviation $(\sigma)$ of the gradients of each parameter sampled from $r$ MOAT trajectories. Analytical model (implemented in [3]) has 9 input parameters (7 material and 2 geometrical parameters) whereas the FEM model (with both inter and intralaminar damage) considers 22 material properties, with open hole tensile strength as the output parameter in both the cases. The input parameters were varied within $\mu \pm 3 \sigma$. Figure 1 (a) and (b) represents the Morris SA plots obtained from the analytical and numerical models, respectively. While the SA combined with analytical model (a total of 200 runs) provides a preliminary understanding of the sensitive parameters, the FEM model based SA (a total of 460 simulations) provides a detailed overview of the key material properties to be considered in an OHT test. Fig 1 (b) shows that the open hole tensile strength is highly dependent on the fiber longitudinal tensile strength $(X T)$, fiber longitudinal tensile fracture toughness $(G X T)$ and cohesive law shape ( $f X T, f G T)$, and the interlaminar fracture toughness in mode II (GHC). In this study, we also consider other configurations such as different laminate sequences, the specimen geometries and the specimen thicknesses to understand their effect on the key parameters.

This work has been accomplished within the framework of an ongoing EU Horizon 2020 Clean Sky 2 Project TREAL (Thermoplastic material allowable generation using a reliability-based virtual modeling platform, Call H2020-CS2-CFP09-2018-02 with Grant agreement ID: 864723) which provided the financial su
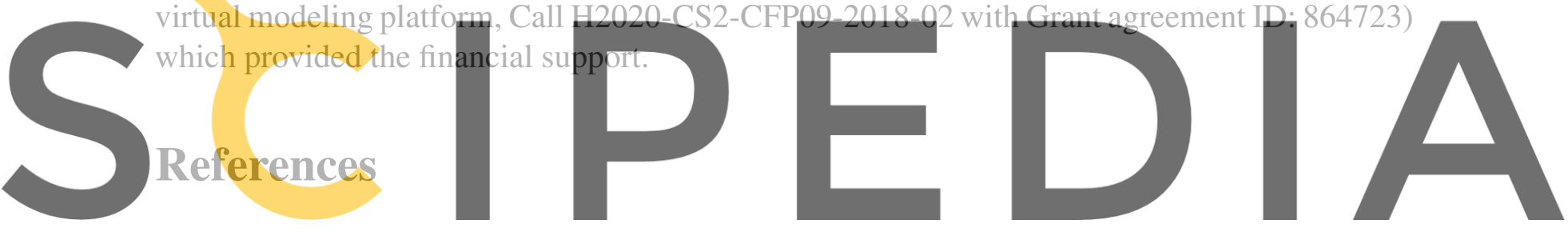

[1] Gan Y, Duan Q, Gong W, Tong C, Sun Y, Chu W, Ye A, Miao C, Di Z. (2014). A compre-

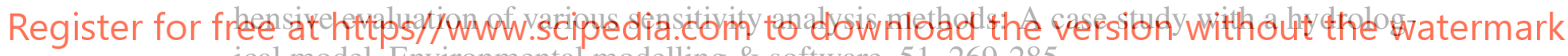
ical model. Environmental modelling \& software. 51, 269-285.

[2] Tian, W. (2013). A review of sensitivity analysis methods in building energy analysis. Renewable and Sustainable Energy Reviews, 20, 411-419.

[3] Furtado, C., Arteiro, A., Bessa, M. A., Wardle, B. L., \& Camanho, P. P. (2017). Prediction of size effects in open-hole laminates using only the Young's modulus, the strength, and the R-curve of the 0 ply. Composites Part A: Applied Science and Manufacturing, 101, 306-317.

[4] Morris, M. D. (1991). Factorial sampling plans for preliminary computational experiments. Technometrics, 33(2), 161-174. 